\title{
The High Amplitude $\delta$ Scuti Variable Star GSC 3109-00162
}

\author{
P. Wils ${ }^{1}$, P. Van Cauteren ${ }^{2,1}$, and R. Groenendaels ${ }^{3}$ \\ ${ }^{1}$ Vereniging Voor Sterrenkunde, Belgium (Patrick.Wils@cronos.be) \\ ${ }^{2}$ Beersel Hills Observatory, Laarheidestraat, 166, 1650 Beersel, Belgium \\ (Paul.VanCauteren@pi.be) \\ ${ }^{3}$ Email: (roger.groenendaels@skynet.be)
}

The ROTSE1 (Robotic Optical Transient Search Experiment 1) survey (Akerlof et al. 2000) found ROTSE1 J183206.54+403555.9 (= GSC 3109-00162; $\left.\alpha_{2000}=18^{\mathrm{h}} 32^{\mathrm{m}} 06^{\mathrm{s}} ; \delta_{2000}=+40^{\circ} 35.9\right)$ to be a $\delta$ Scuti variable with a period of 0.102145 in the approximate magnitude range $12.4-12.9$.

We observed the star on seven nights between June and August 2001, obtaining 1045 data points, totaling 18.2 hours of photometry. The instruments used were a 0.40-m telescope, equipped with a ST7E CCD camera (PVC: 16.1 hours of photometry), and a 0.30-m telescope, equipped with a Hisis24 CCD camera (RG: 2.1 hours). No filter was used. The exposure times varied between 50 and 60 seconds. The images were reduced with the aperture photometry procedure of the Mira AP software package (produced by Axiom Research Inc.).

The brightness of the variable was measured with respect to GSC 310902150, while GSC 3109-01092 served as check star. To determine the best comparison star we also collected some images in B and V light, using a filterset following Bessell's specifications. From these we derived instrumental $\Delta(B-V)$ values with respect to the variable star. Our comparison star was chosen on basis of the smallest possible colour difference (in this case $\Delta(B-V)=0.09$ mag for the comparison star and $\Delta(B-V)=0.26$ mag for the check star) in order to reduce the influence of the differential colour term coupled to the second order extinction coefficient. The standard deviation of the differential magnitudes between the comparison and check star was of the order of 0 . 006 (with nightly variations between $0 .{ }^{\mathrm{m}} 004$ and $0 .{ }^{\mathrm{m}} 009$ depending on sky conditions). 
Table 1: Times of maxima from the CCD observations

\begin{tabular}{|lrcc|}
\hline JD Hel. & E & O-C & Observer \\
\hline 2451243.6018 & -8232 & 0.0006 & ROTSE1 \\
2452084.4973 & 0 & -0.0002 & PVC \\
2452085.4158 & 9 & -0.0010 & PVC \\
2452132.4054 & 469 & -0.0003 & PVC \\
2452132.5081 & 470 & 0.0002 & PVC \\
2452136.4919 & 509 & 0.0002 & RG \\
2452138.4320 & 528 & -0.0005 & PVC \\
2452145.4814 & 597 & 0.0005 & PVC \\
2452150.3847 & 645 & 0.0006 & PVC \\
\hline
\end{tabular}

In Table 1 we list the times of maxima that were determined from the new observations $((\mathrm{O}-\mathrm{C})$ values are listed with respect to the ephemeris derived below). The table also contains one time of maximum derived from a phase diagram of the ROTSE1 data (available through http://www.umich.edu/ rotse). The number of cycles between the ROTSE1 maximum and our data can be determined unambiguously, leading to the improved ephemeris:

$$
\begin{gathered}
\text { Max. = HJD } 2452084.4974+0.1021497 \times E . \\
\pm 0.0003 \pm 0.0000002
\end{gathered}
$$

Figure 1 shows the phased light curve from the data obtained by PVC.

Our results thus confirm that GSC 3109-00162 is a high amplitude $\delta$ Scuti star (HADS) with an unfiltered CCD amplitude of $0.55 \mathrm{mag}$. The rise from minimum to maximum (M-m) takes 0.31 cycles. No trace of multiperiodicity was found, although there may be small differences in the height of the maxima.

Acknowledgments. We thank Dr. Patricia Lampens, Koninklijke Sterrenwacht van België, for helpful comments. P. Van Cauteren is grateful to the Royal Observatory of Belgium for putting at his disposal material acquired by project G.0265.97 of the Fund for Scientific Research (FWO) - Flanders (Belgium). 


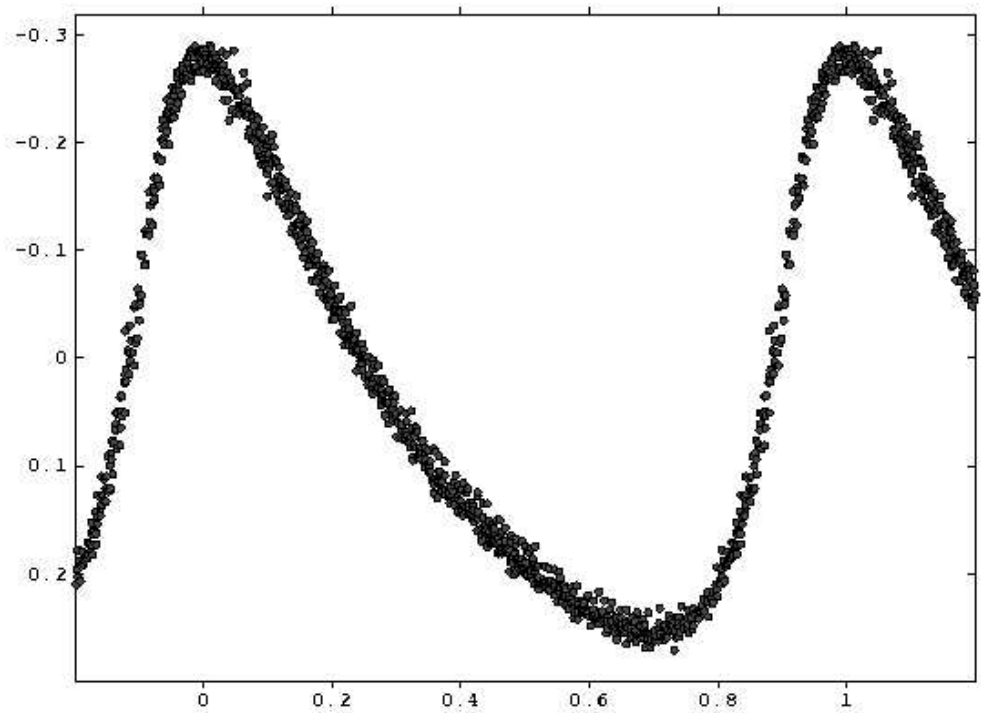

Figure 1: Phase diagram for GSC 3109-00162.

\section{References}

Akerlof, C., Amrose, S., Balsano, R., Bloch, J., Casperson, D., Fletcher, S., Gisler, G., Hills, J., Kehoe, R., Lee, B., Marshall, S., McKay, T., Pawl, A., Schaefer, J., Szymanski, J., Wren, J. 2000, AJ 119, 1901 